This document is the accepted manuscript version of the following article:

Stefani, D., Perrin, M., Gutiérrez-Cerón, C., Aragonès, A. C., Labra-Muñoz, J., Carrasco, R. D. C., ... Hi11, J. P. (2018). Mechanical tuning of through-molecule conductance in a conjugated calix[4]pyrrole. Chemistry select, 3(23), 6473-6478. https://doi.org/10.1002/s1ct.201801076

DOI: $10.1002 /$ slct.201801076

Full Paper

Organic \& Supramolecular Chemistry

Received: $12 .^{\wedge}$ April.^2018

Accepted: 6.^June.^2018

\title{
Mechanical Tuning of Through-Molecule Conductance in a Conjugated Calix[4]pyrrole
}

Davide Stefani, ${ }^{[\mathrm{a}]}$ Dr. Mickael Perrin, ${ }^{+[\mathrm{a}]}$ Cristian Gutierréz-Cerron, ${ }^{[\mathrm{b}]}$ Albert C. Aragonès, Jacqueline Labra-Muñoz, ${ }^{[\mathrm{b}]}$ Rodrigo D. C. Carrasco, ${ }^{[\mathrm{b}]}$ Dr. Yoshitaka Matsushita, ${ }^{[\mathrm{d}]}$ Dr.

Zdenek Futera, ${ }^{[\mathrm{e}]}$ Dr. Jan Labuta, ${ }^{[\mathrm{f}]}$ Dr. Thien H. Ngo, ${ }^{[\mathrm{f}]}$ Prof. Katsuhiko Ariga, ${ }^{[\mathrm{f}]}$ Prof. Ismael Díez-Pérez, ${ }^{\#[c]}$ Prof. Herre ${ }^{\wedge \wedge}$ S.J. van ${ }^{\wedge \wedge}$ der Zant, ${ }^{[a]}$ Prof. Diana Dulić, ${ }^{[b]}$ and Dr. Jonathan P. Hillo000-0002-4229-5842*[f]

[a] <orgDiv/>Kavli Institute of Nanoscience, <orgName/>Delft University of Technology, <street/>Lorentzweg 1, <postCode/>2628 CJ Delft, <country/>The Netherlands

[b] <orgDiv/>Physics Department, <orgDiv/>Faculty of Physical and Mathematical Sciences, <orgName/>University of Chile, Av. Blanco Encalada 2008, $<$ country/>Santiago Chile E-Mail: ddulic@ing.uchile.cl

[c] <orgDiv/>Department of Materials Science and Physical Chemistry, <orgDiv/>Institute for Bioengineering of Catalonia (IBEC) \& Institute of Theoretical and Computational Chemistry, <orgName/>University of Barcelona, Martí i Franquès 1, <postCode/>Barcelona 08028, <country/>Spain

[d] <orgDiv/>Research Network and Facilities Division, <orgName/>National Institute for Materials Science, Sengen 1--2-1, Tsukuba, <postCode/>Ibaraki 305--0047, $<$ country/>Japan 
[e] <orgDiv/>School of Chemical \& Bioprocess Engineering, <orgName/>University College Dublin, Belfield, <city/>Dublin 4, <country/>Ireland

[f] <orgDiv/> WPI Center for Materials Nanoarchitectonics, <orgName/>National Institute for Materials Science, Namiki 1--1, Tsukuba, <postCode/>Ibaraki 305--0044, $<$ country/> Japan

E-Mail: Jonathan.Hill@ @ims.go.jp

[+] Present address: Empa, Swiss Federal Laboratories for Material Science and Technology, Transport at Nanoscale Interfaces Laboratory, CH-8600 Dübendorf, Switzerland

\# Present address: Department of Chemistry, King's College London, Britannia House, 7 Trinity Street, London SE1 1DB, U.K.

<pict> Supporting information for this article is available on the WWW under <url>http://dx.doi.org/10.1002/slct.201801076</url>

calix[4]pyrrole

mechanically-controlled break junction

oxoporphyrinogen

single molecule

Pulling a tetrapyrrole. A conformationally flexible calix[4]pyrrole possessing a conjugated electronic structure was used to investigate the influence of mechanical stretching on the single-molecule conductance of these molecules using the mechanically-controlled break junction technique.

A conformationally flexible calix[4]pyrrole possessing a conjugated electronic structure (an $\mathrm{N}$-substituted oxoporphyrinogen (OxP) related to porphyrin) was used to investigate the influence of mechanical stretching on the single-molecule conductance of these molecules using the mechanically-controlled break junction (MCBJ) technique. The results show that the molecule can be immobilized in a single-molecule break junction configuration, giving rise to different behaviours. These include step-like features in the conductance vs. displacement 
traces as well as conductance traces that exhibit a slower decay ('downhill' traces) than measured for direct tunneling. The latter class of traces can be associated with the mechanical manipulation (i.^e., stretching) of the molecule with inter-electrode distances as long as $2^{\wedge \wedge} \mathrm{nm}$. Density functional theory (DFT) calculations reveal that OxP molecules are stable under stretching in the length regime studied.

\section{Introduction}

Molecular nanotechnology has introduced a requirement for better understanding of how single molecules behave under different applied stimuli including electrical ${ }^{[1-4]}$ and/or magnetic fields, ${ }^{[5-7]}$ incident light ${ }^{[8]}$ (i. ${ }^{\wedge}$., an optical field), mechanical manipulation, ${ }^{[9--11]}$ or combinations of these. These interactions with external stimuli have been studied for molecules contained in solution, ${ }^{[12,13]}$ at interfaces,${ }^{[14,15]}$ for individual molecules manipulated

a)
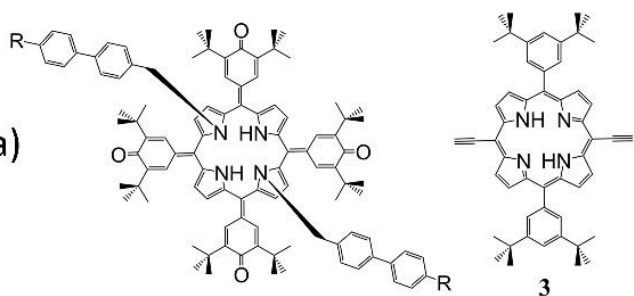

$\mathrm{R}=-\mathrm{SCH}_{3}(\mathbf{1}) ;-\neg-\neg \mathrm{SCH}_{3}(2)$

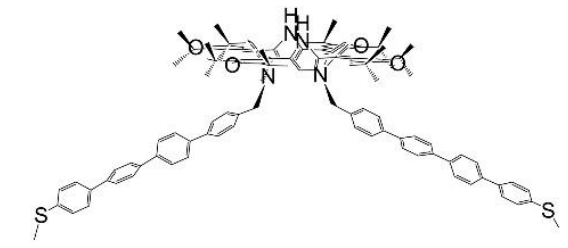

b)

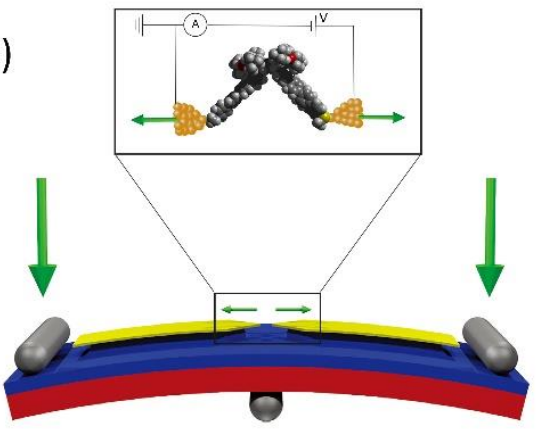

using a scanning tunneling microscope tip ${ }^{[16,17]}$ or fixed in mechanically controlled break junction (MCBJ) configurations. ${ }^{[18]}$ 
Porphyrins are interesting compounds for such studies and we $\mathrm{e}^{[19--21]}$ and others ${ }^{[22,23]}$ have investigated their properties when assembled into single-molecule devices. Concerning
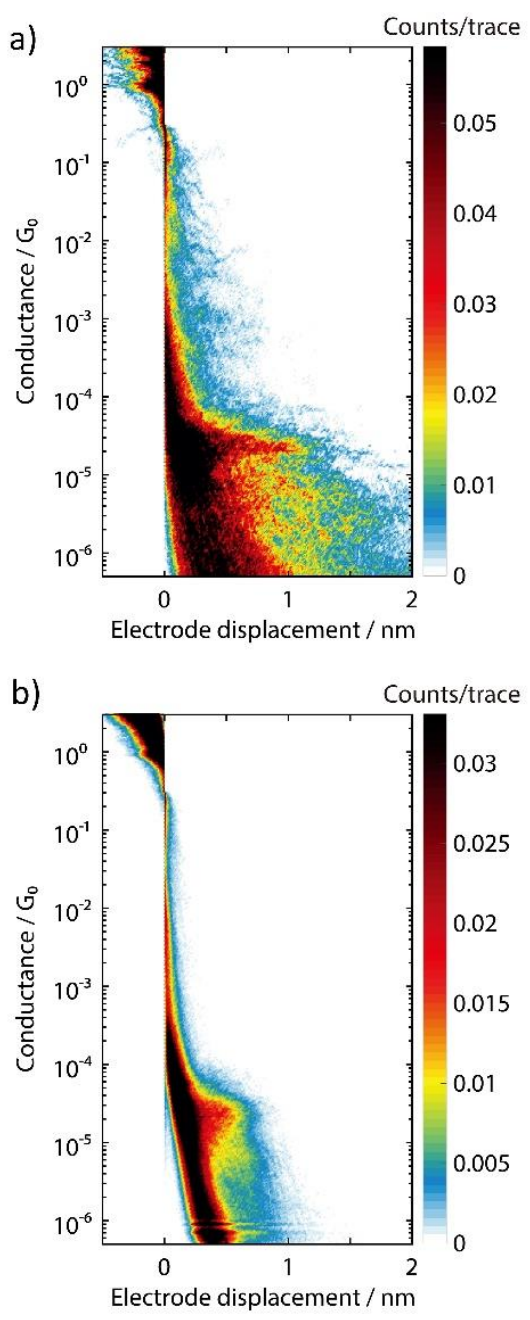

in-situ mechanical manipulations, porphyrins, such as functionalized meso-tetraphenylporphyrins, while possessing some torsional flexibility especially about the meso-phenylporphyrin bonds, are not suitable for studies in the nanometre regime. In this respect $\mathrm{N}$ alkylated oxoporphyrinogens $\left(\mathrm{OxP} ;{ }^{[24]}\right.$ Figure $^{\wedge \wedge} 1<$ figr1 $\left.>\right)$ are promising candidates because the calix[4]pyrrole core contained in these molecules is known to be sufficiently flexible with respect to the conformation of its pyrrole groups. ${ }^{[25-28]}$ Calix[4]pyrrole is known to exist in different forms including saddle (1,2-alternating) and cone conformations although the latter cone forms have not been reported for OxP-type macrocycles. ${ }^{[29]}$ Various other studies have revealed that some molecules can be mechanically twisted or stretched and that these changes can be coupled not only to its conductance but also to more exotic phenomena such as a spin crossover transition. ${ }^{[10]}$ The application of mechanical stress to rigid molecules such as 
benzene dithiols or other inflexible molecules has been suggested as a means of controlling the electronic properties of single-molecule junctions. ${ }^{[30,31]}$ On the other hand, deliberate use of molecules that can be distorted for these purposes is less common. ${ }^{[32--35]}$

In this work, using the mechanically controlled break junction (MCBJ) technique, we investigated the low-bias electrical conductance of molecules 1--3 (Figure ${ }^{\wedge \wedge} 1<x$ figr $\left.1>a\right)$ based on the calix[4]pyrrole core ${ }^{[36,37]}$ addressing the possibility of operating the ostensibly flexible OxP molecule as a 'hinge'-like mechanical potentiometer (i.^e. a variable resistance device that can be used to control current). The OxP molecular system is attractive from this point-of-view because its incorporation into more complex molecular systems is a relatively simple matter, ${ }^{[38,39]}$ and it has the property of being able to accommodate guests (e. ${ }^{\wedge} \mathrm{g}$. analytes) through hydrogen bonding interactions. The chemistry of these molecules has been intensively investigated. ${ }^{[25--28,40<\mathrm{M}->43]}$ and their properties include various sensing activities ${ }^{[44-}$ ${ }^{-47]}$ due to their intense colors presented by the conjugation of the calix[4]pyrrole core with hemiquinonoid meso-substituents.

\section{Results and Discussion}

Figure $^{\wedge \wedge} 1<x$ figr $1>$ a shows the chemical structure of OxP molecules 1--3. Compounds $\mathbf{1}$ and $\mathbf{2}$ are subject of this study while $\mathbf{3}$ was used as a rigid control reference for the measurements reported here. ${ }^{[48]}$ To improve the chances of the binding processes, molecules are required to be terminated with appropriate anchoring groups such as the thiomethyl groups in $\mathbf{1}$ and $\mathbf{2}$ or ethynylene in $\mathbf{3}$. The conductances of $\mathbf{1 - - 3}$ were measured in an MCBJ setup in air at room temperature. Details about the MCBJ setup and the measuring technique have been described elsewhere. ${ }^{[49.50]}$ A solution with the molecules under investigation was prepared by dissolving the starting compound in chloroform with a few drops of trifluoroacetic acid to increase the solubility. A $2 \mu \mathrm{L}$ droplet of the solution was then dropcast onto the newly characterized device. Large numbers of consecutive breaking traces collected from individual junctions constitute a set which is used to construct a $2 \mathrm{D}$ histogram of the conductance (conductance versus electrode displacement). ${ }^{[51]}$ By integrating over the 
displacement, a one-dimensional histogram is obtained, from which the most probable junction conductance can be estimated (see Figures ${ }^{\wedge} \mathrm{S} 2 \mathrm{c}$ and $\mathrm{S} 3 \mathrm{c}$ ).

Interestingly, the shorter OxP molecule $\mathbf{1}$ is difficult to incorporate into the break junction. Multiple experiments were performed on 1, but none yielded clear conductance peaks in the histograms (see Figure^^${ }^{\wedge} 1$ ). The use of the extended derivative $\mathbf{2}$ allowed a significantly enhanced yield of molecule occupation in the break junction experiments probably due to longer separation of the ligating thiomethyl groups and an increasingly linear form of the molecule compared to $\mathbf{1}$. In addition, the longer $\mathrm{N}$-substituents minimize steric effects between the OxP chromophore and the substrate, which might obstruct binding.

Figure $^{\wedge \wedge} 2<$ figr $2>$ a shows the $2 \mathrm{D}$ histogram constructed from 1,650 consecutive breaking traces recorded after drop-casting a solution containing OxP molecule 2. Inspecting the individual traces, a large percentage of them (30.4\%) exhibit a slow decrease in conductance upon increasing electrode separation; this has been colloquially named
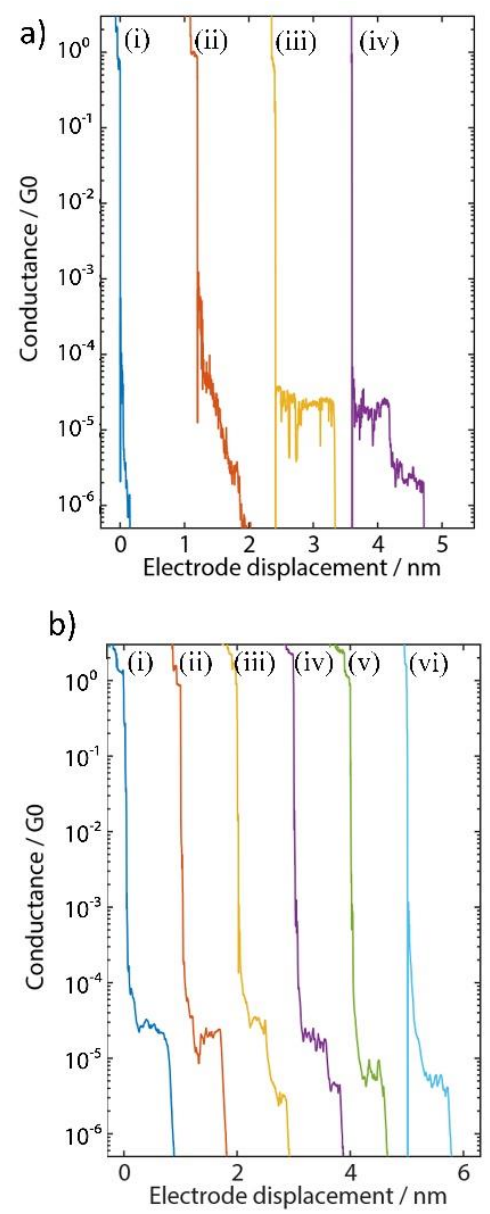

'downhill' behaviour (Figure^^3<figr3>(a)). These traces can be distinguished from those in 
which no molecule was contacted from their trace length, which is generally longer than $0.5^{\wedge \wedge} \mathrm{nm}$ and can be as long as more than $2^{\wedge \wedge} \mathrm{nm}$ (see Figure ${ }^{\wedge} 3<\mathrm{xfigr} 3>\mathrm{a}(\mathrm{ii}-\mathrm{iii})$ ). For comparison, empty junctions may also exhibit 'downhill' behaviour but their length is typically less than $0.5^{\wedge \wedge} \mathrm{nm}$ (see Figure ${ }^{\wedge} 3<\mathrm{xfigr} 3>\mathrm{a}(\mathrm{i})$ ). Furthermore, by considering the tunnelling decay constant $(\beta)$, there is a clear distinction between empty junctions (tunneling through vacuum, $\beta=-6.5)$ and downhill traces $(\beta \geq<M->3)$ (see Supporting Information). Some traces show plateaus in conductance, as displayed by Figure ${ }^{\wedge} 3<x f i g r 3>a(i i i-i v) . ~ T o$ accentuate these plateaus we employed a selection criterion and focused our analysis only on those traces with high counts in the $1 \times 10^{<\mathrm{M}->4}-1 \times 10^{<\mathrm{M}->6} \mathrm{G}_{0}$ region (for details see the Supporting Information). We find peaks in the one-dimensional conductance histogram around $2.3 \times 10^{<\mathrm{M}->5} \mathrm{G}_{0}, 6.6 \times 10^{<\mathrm{M}->6} \mathrm{G}_{0}$ and $2.1 \times 10^{<\mathrm{M}->6} \mathrm{G}_{0}$. Further experimental details are given in the Supporting Information.

Figure $^{\wedge \wedge} 2<x$ figr $2>b$ shows the $2 \mathrm{D}$ histogram constructed from 10,000 traces recorded after drop-casting a solution containing rigid control compound 3. It shows a high count region around $2 \times 10^{<\mathrm{M}->5} \mathrm{G}_{0}$ that extends for less than $1^{\wedge \wedge} \mathrm{nm}$. The breaking traces of molecular junctions exhibit mostly flat plateaus and no significant variation in conductance upon stretching. For this molecule no traces were found showing a 'downhill'-like behaviour (Figure ${ }^{\wedge} 3<x$ figr3>(b)) as was observed for the OxP molecule 2. After selecting traces with high counts in the $\left[5 \times 10^{<\mathrm{M}->5}-5 \times 10^{<\mathrm{M}->6}\right] \mathrm{G}_{0}$ region (see Supporting Information for details), we find two plateaus in conductance, one around $2.1 \times 10^{<\mathrm{M}->5} \mathrm{G}_{0}$ and one at $5.9 \times$ $10^{<\mathrm{M}->6} \mathrm{G}_{0}$, albeit with fewer counts. Overall, the results of MCBJ measurements can be summarized qualitatively for each compound as: $\mathbf{1}$ - low yield of junction formation; no clear conclusions about the presence of the molecules can therefore been drawn; 2 - high yield of junctions with large proportion of 'downhill' traces; $\mathbf{3}$ - junctions which mostly exhibit flat plateaus with little or no variation in conductance on stretching.

We will now discuss the downhill behaviour observed in breaking traces of 2 . There are several possible origins for the observation of downhill conductance behaviour in these systems. As suggested by Nuckolls et ${ }^{\wedge \wedge}$ al.,${ }^{[52]}$ one possible explanation for this behaviour is the injection of charge directly into the molecules' $\pi$-electronic system while the contacts are 
sliding along the electrodes. This effect was suggested, among other reasons, by a jump in the conductance trace as the current began flowing through the whole molecule (injection through the anchoring). In the case of OxP molecule 2, such jumps in conductance were not observed, which in turn suggests that the observed variations in conductance should have a different origin. The flexibility of the calix[4]pyrrole macrocycle could play a key role in this, as the variation in the dihedral angles subtended between opposing pyrrole groups of $\mathrm{OxP}$, which changes as the molecule is stretched across the junction, could be the main origin of 'downhill' traces. ${ }^{[53,54]}$ This interpretation is corroborated by the fact that 'downhill' conductance traces are observed for the flexible 2 but not for the rigid reference molecule $\mathbf{3}$. If we consider the calix[4]pyrrole unit contained in the OxP molecules from an X-ray crystallographic perspective, then we find that variation in the conformation of the tetrapyrrole core can be caused by differences in the substitution patterns of the compounds shown in Figure ${ }^{\wedge} 4<$ figr $4>$ a. ${ }^{[25--28]}$ In Figure ${ }^{\wedge \wedge} 4<x$ figr $4>$, values of $\alpha$ denote the dihedral angle subtended between the planes of $\mathrm{N}$-substituted pyrrole groups.

Thus, the introduction of different $\mathrm{N}$-substituents leads to a variation in the dihedral

a)

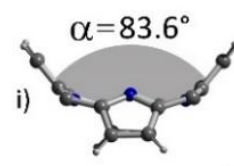

ii)
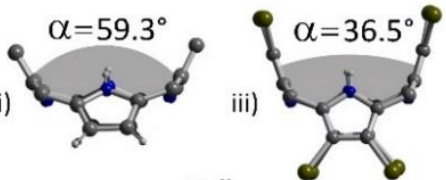

b)
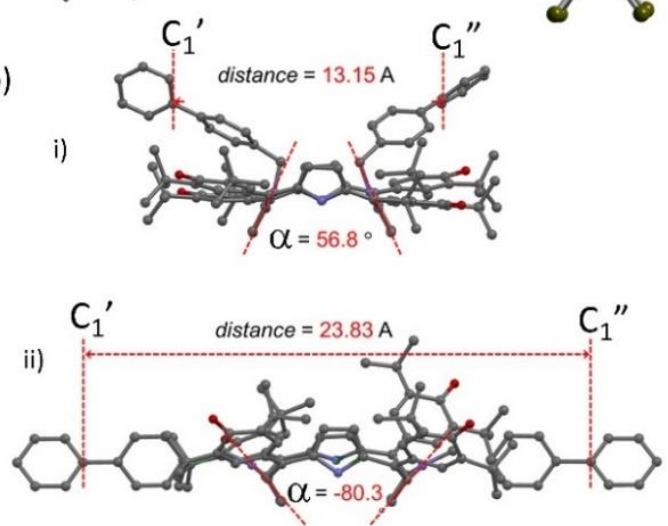

a)

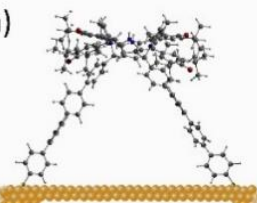

b)
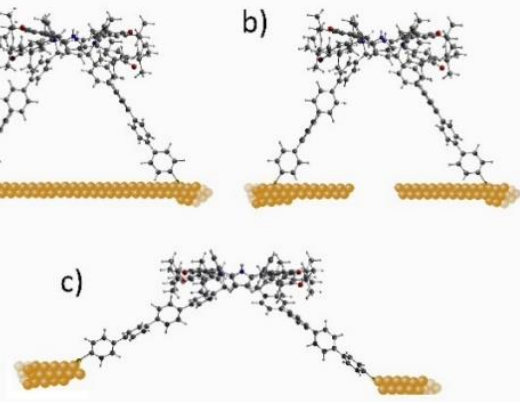

d)

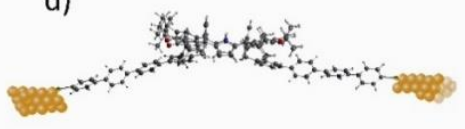

angle subtended between opposing pyrrole groups, as does changing the substituents at pyrrole $\beta$-positions (in this case bromine atoms). The dihedral angle subtended between opposing pyrrole groups of OxP varies between $83.6^{\circ}$ (for an unsubstituted derivative) down to $36.5^{\circ}$ (for a highly substituted derivative containing $\mathrm{N}$-substituents and bromine atoms at pyrrole groups) establishing the flexibility of the OxP framework. 
It should be noted that, notwithstanding identity of $\beta$-substituents, compounds containing similar benzylic substituents have similar values of $\alpha$ (for example, the DFT calculated structure of $\mathrm{N}_{21} \mathrm{~N}_{23}$-bis(4-phenylbenzyl)-OxP has $\alpha=56.8^{\circ}$, which is similar to that observed for the X-ray crystal structure of $\mathrm{N}_{21} \mathrm{~N}_{23}$-bis(4-bromobenzyl)-OxP, $\alpha=59.3^{\circ}$ ). We have investigated this point further by performing DFT calculations ${ }^{[55]}$ on the OxP molecule

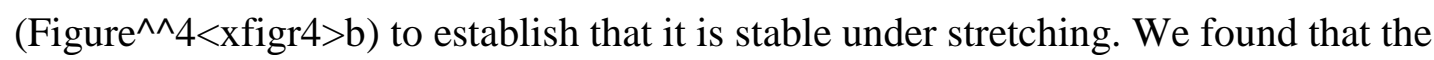
molecule is computationally stable (using wB97XD/cc-pVDZ for full geometry optimization with fixed biphenyl distance) even under deformation beyond the sub-nanometer-scale stretching length typical of MCBJ experiments. Starting from a conformation similar to that of the 4-bromobenzyl-OxP precursor, optimization was performed with different distances fixed between the N-substituents of OxP. A selected stretched conformation is shown in Figure ${ }^{\wedge} 4<x f i g r 4>b(i i)$ revealing a possible extension of around $1^{\wedge \wedge} \mathrm{nm}$. It is notable that the stretched conformations of $\mathrm{N}$-substituted OxP remain at least chemically stable far beyond the crystallographically observed minimum of pyrrole group dihedral angle $\left(\sim 37^{\circ}\right)$. The high yield of 'downhill' conductance traces obtained for 2 can thus be seen as an indication of the molecules' flexibility based on conformational variation at the calix[4]pyrrole core with typical extension of $1^{\wedge \wedge} \mathrm{nm}$ extending as far as $2^{\wedge \wedge} \mathrm{nm}$. This corresponds with the dimensions and possible maximum extension of the molecule where the stretched form could be stable according to DFT energy minimization (see Figure ${ }^{\wedge} 5<$ figr $5>$ ). Junctions containing 2 occasionally showed plateaus in conductance rather than 'downhill' behaviour suggesting the possibility of forming molecular junctions with configurations that do not involve the intended anchoring groups.

\section{Conclusions}

In conclusion, we have demonstrated mechanical modulation of single molecule conductance in the OxP molecular system using the mechanically-controlled break junction set-up. Long, slow decaying conductance traces ('downhill') were observed. The flexibility of the calix[4]pyrrole core contained in the molecules used and, in particular, the hinge-like opening of $\mathrm{OxP}$ due to the possible variability of dihedral angle between opposing pyrrole groups of the structure suggest that this phenomenon is due to the stretching deformation of 
the molecule. The known X-ray crystallographic features of OxP derivatives corroborate this theory, with DFT calculations indicating that the OxP macrocycle can be extended on a scale corresponding with that of the measurements. The ultimate aim of incorporating such molecules into a device is to study how potential supramolecular interactions occurring at the calix[4]pyrrole core can further influence the properties of MCBJ structures, and accounts of these matters will appear later.

\section{Supporting Information Summary}

Supporting information contains the following information: general methods; details of syntheses of $\mathbf{1}$ and 2; experimental details of MCBJ, X-ray crystallography and computational methods; statistical analysis of MCBJ data, NMR and mass spectra of $\mathbf{1}$ and 2.

\section{Acknowledgements}

This study was supported by <cgs $>$ JSPS KAKENHI $</$ cgs $>$ Grant Numbers $<$ cgn $>15^{\wedge \wedge}$ K13684</cgn $>$ and $<$ cgn>JP16H06518</cgn>, FONDECYT Regular Grant 1140770, The Dutch funding agencies FOM and NWO/OCW, by the EU through a RISE(DAFNEOX) project, SEP 210165479 and an advanced ERC grant (Mols@ Mols); device fabrication was done at the Kavli Nanolab at Delft. This work was partly supported by World Premier International Research Center Initiative (WPI Initiative), MEXT, Japan, the Core Research for Evolutional Science and Technology (CREST) program of Japan Science and Technology Agency (JST), Japan.

\section{Conflict of Interest}

The authors declare no conflict of interest.

$<$ lit1 ><jnl>C. Baldacchini, V. Kumar, A.^^R. Bizzari, S. Cannistraro, Appl. Phys. Lett. 2015, $106,183701 / 1-4</ \mathrm{jnl}>$.

$<$ lit2 $><$ jnl $>$ G. ${ }^{\wedge \wedge}$ D. Harzmann, R. Frisenda, H.^^${ }^{\wedge} .^{\wedge \wedge} \mathrm{J}$. van der Zant, M. Mayor, Angew.

Chem. Int. Ed. 2015, 54, 13425--13430</jnl>.

$<$ lit3 $><$ jnl $>$ M. Urdampilleta, S. Klayatskaya, M. Ruben, W. Wernsdorfer, ACS Nano 2015, 9, 4458--4464</jnl >

<lit4><jnl>I. Diez-Perez, Z. Li, J. Hihath, J. Li, C. Zhang, X. Yang, L. Zang, Y. Dai, X. Feng, K. Müllen, N. Tao, Nat. Commun. 2010, 1, 31</jnl>. 
$<$ lit5><jnl>R. Hayakawa, M.^^A. Karimi, J. Wolf, T. Huhn, M.^^S. Zöllner, C. Herrmann, E. Scheer, Nano Lett. 2016, 16, 4960--4967</jnl>.

$<$ lit6><jnl>B. Warner, F. El Hallak, H. Prüser, J. Sharp, M. Persson, A.^^J. Fischer, C.^^F. Hirjibehedin, Nat. Nanotechnol. 2014, 10, 259--263</jnl>.

$<$ lit7><jnl>A.^^C. Aragones, D. Aravena, J.^^I. Cerda, Z. Acis-Castillo, H. Li, J.^^A. Real, F. Sanz, J. Hihath, E. Ruiz, I. Díez-Pérez, Nano Lett. 2016, 16, 218--226</jnl>.

$<$ lit8 $><j n l>N$. Darwish, A. ${ }^{\wedge}$ C. Aragones, T. Darwish, S. Ciampi, I. Díez-Pérez, Nano Lett. 2014, 14, 7064--7070</jnl>.

$<$ lit9><jnl>Y. Kitaguchi, S. Habuka, H. Okuyama, S. Hatta, T. Aruga, T. Frederiksen, M.

Paulsson, H. Ueba, Beilstein J. Nanotechnol. 2015, 6, 2088--2095</jnl>.

$<$ lit10 $><$ jnl $>$ R. Frisenda, G. ${ }^{\wedge}$ D. Harzmann, J.^^A.^^C. Gil, J.^^M. Thijssen, M. Mayor, H.^^S.^^J. van der Zant, Nano Lett. 2016, 16, 4733--4737</jnl>.

$<$ lit11><jnl>I. Diez-Perez, J. Hihath, T. Hines, Z.-S. Wang, G. Zhou, K. Müllen, N. Tao, Nat. Nanotechnol. 2011, 6, 226--231</jnl>.

$<$ lit12><jnl >A. Faulkner, T. Van Leeuwen, B. ${ }^{\wedge}$ L. Feringa, S. ${ }^{\wedge}$ J. Wezenberg, J. Am. Chem. Soc. 2016, 138, 13597--13603</jnl .

$<$ lit13><jnl>S. Wiedbrauk, B. Maerz, E. Samoylova, A. Reiner, F. Trommer, P. Mayer, W.

Zinth, H. Dube, J. Am. Chem. Soc. 2016, 138, 12219--12227</jnl>.

$<$ lit14><jnl>J. Schutte, R. Bechstein. P. Rahe, H. Langhals, M. Rohlfing, A. Kuhnle, Nanotechnology 2011, 22, 245701</jnl>.

$<$ lit15><jnl>B.^^J. Heinrich, L. Braun, J.^^I. Pascal, K. Franke, J. Nano Lett. 2015, 15, 4024$-4028</$ jnl $>$.

$<$ lit16><jnl >P. Liljeroth, Nat. Nanotechnol. 2012, 7, 5--6</jnl >.

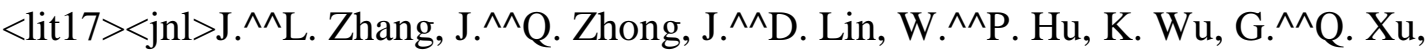

A.^^T.^^S. Wee, Chen, W. Chem. Soc. Rev. 2015, 44, 2998--3022</jnl>.

$<$ lit18><jnl>D. Xiang, H. Jeong, T. Lee, D. Mayer, Adv. Mater. 2013, 25, 4845--4867</jnl>. 
$<$ lit19><jnl $>$ M. ${ }^{\wedge}$ L. Perrin, F. Prins, C.^^A. Martin, A. ${ }^{\wedge}$ J. Shaikh, R. Eelkema, J.^^H. van Esch, T. Briza, R. Kaplanek, V. Kral, J.^^M. van Ruitenbeek, H.^^S.^^J. van der Zant, D. Dulić, Angew. Chem. Int. Ed. 2011, 50, 11223--11226</jnl>.

$<$ lit20><jnl $>$ M.^^.$^{\wedge}$. Perrin, C. ${ }^{\wedge} \mathrm{J} .{ }^{\wedge \wedge} \mathrm{O}$. Verzijl, C. ${ }^{\wedge}$ A. Martin, A..$^{\wedge} \mathrm{J}$. Shaikh, R. Eelkema, J. ${ }^{\wedge} H$. van Esch, J.^^M. van Ruitenbeek, J.^^M. Thijssen, H.^^S.^^J. van der Zant, D. Dulić, Nat. Nanotechnol. 2013, 8, 282--287</jnl>.

$<$ lit21 ><jnl>A.^^C. Aragonès, N. Darwish, W.^^J. Saletra, L. Pérez-García, F. Sanz, J. Puigmartí-Luis, D.^^B. Amabilino, I. Díez-Pérez, Nano Lett. 2014, 14, 4751-$4756</$ jnl $>$.

$<$ lit22><jnl>Z.-F. Liu, S. Wei, H. Yoon, O. Adak, I. Ponce, Y. Jiang, W.-D. Jang, L.^^M.

Campos, L. Venkataraman, J.^^B. Neaton, Nano Lett. 2014, 14, 5365--5370</jnl>. $<$ lit23><jnl >Z. Li, E. Borquet, J. Am. Chem. Soc. 2012, 134, 63--66</jnl>. $<$ lit24><other > OxP is an abbreviated form of 5,10,15,20-(3,5-di-t-butyl-4-oxocyclohexa-2,5dienylidene)porphyrinogen</other>. $<$ lit25><jnl>A.^^J. Golder, L. ${ }^{\wedge}$ R. Milgrom, K. ${ }^{\wedge}$ B. Nolan, D. ${ }^{\wedge}$ C. Povey, J. Chem. Soc., Chem. Comm. 1989, 1751--1753</jnl >.

$<$ lit26><jnl>J.^^P. Hill, W. Schmitt, A.^^L. McCarty, K. Ariga, F. D’Souza, Eur. J. Org. Chem. 2005, 2893--2902</jnl >. $<$ lit27><jnl>J.^^P. Hill, I.^^J. Hewitt, C.^^E. Anson, A.^^K. Powell, A.^^L. McCarthy, P.^^A. Karr, M.^^E. Zandler, F. D’Souza, J. Org. Chem., 2004, 69, 5861--5869</jnl>. $<$ lit28><jnl>W.^^A. Webre, J.^^P. Hill, Y. Matsushita, P.^^A. Karr, K. Ariga, F. D’Souza, Dalton Trans. 2016, 45, 4006--4016</jnl>.

$<$ lit29><jnl>C.^^J. Woods, S. Camiolo, M.^^E. Light, S.^^J. Coles, M.^^B. Hursthouse, M.^^A. King, P.^^A. Gale, J.^^W. Essex, J. Am. Chem. Soc. 2002, 124, 8644-$8652</$ jnl $>$.

$<$ lit30 $><$ jnl $>$ C. Bruot, J. Hihath, N.^^J. Tao, Nat. Nanotechnol. 2012, 7, 35--40. $\quad</$ jnl $>$ 
$<$ lit31 ><jnl>D. Xiang, Y. Zhang, F. Pyatkov, A. Offenhäusser, D. Mayer, Chem. Commun, 2011, 47, 4760--4762</jnl >.

$<$ lit32><jnl>I. Franco, C.^^B. George, G. ${ }^{\wedge}$ C. Solomon, G. ${ }^{\wedge}$ C. Schatz, M. ${ }^{\wedge}$ A. Ratner, $J$. Am. Chem. Soc. 2011, 133, 2242--2249</jnl>.

$<$ lit33><jnl>I. Franco, C.^^B. George, G. ${ }^{\wedge}$ C. Solomon, G. ${ }^{\wedge}$ C. Schatz, M. ${ }^{\wedge \wedge}$ A. Ratner, $J$. Am. Chem. Soc. 2011, 133, 15714--15720</jnl>.

$<$ lit34><jnl>L. Lafferentz, F. Ample, H. Yu, S. Hecht, C. Joachim, C. Grill, Science 2009, $323,1193--1197</ j n l>$.

$<$ lit35><jnl>A. Pirrotta, L. De Vico, G. ${ }^{\wedge}$ C. Solomon, I. Franco, J. Chem. Phys. 2017, 146, $092329</$ jnl $>$.

$<$ lit36><jnl>P.^^A. Gale, J.^^L. Sessler, V. Kral, Calixpyrroles. Chem. Commun. 1998, 1-$8</$ jnl $>$.

$<$ lit37><jnl>J.^^L. Sessler, P. Anzenbacher, K. Jursikova, H. Miyaji, J.^^W. Genge, N.^^A.

Tvermoes, W.^^E. Allen, J.^^A. Shriver, P.^^A. Gale. V. Kral, Pure Appl. Chem. 1998, 70, 2401--2408</jnl >.

$<$ lit38><jnl>F. D’Souza, N.^^K. Subbaiyan, Y. Xie, J.^^P. Hill, K. Ariga, K. Ohkubo, S.

Fukuzumi, J. Am. Chem. Soc. 2009, 131, 16138--16146</jnl>.

$<$ lit39><jnl>W.^^A. Webre, H.^^B. Gobeze, S. Shao, P.^^A. Karr, K. Ariga, J.^^P. Hill, F.

D’Souza, Chem. Commun. 2018, 54, 1351--1354</jnl>.

$<$ lit40><jnl>L.^^R. Milgrom, J.^^P. Hill, W.^^D. Flitter, J. Chem. Soc., Perkin Trans. 2

1994, 521--524</jnl >.

$<$ lit41><jnl>L.^^R. Milgrom, J.^^P. Hill, G. Yahioglu, J. Heterocyclic Chem. 1995, 32, 97-$101</$ jnl $>$.

$<$ lit42><jnl>L.^^R. Milgrom, J.^^P. Hill, P.^^F. Dempsey, Tetrahedron 1994, 50, 13477-$13484</ \mathrm{jnl}>$. 
$<$ lit43><jnl>Y. Xie, J.^^P. Hill, A. ${ }^{\wedge}$ L. Schumacher, A. ${ }^{\wedge}$ S. ${ }^{\wedge}$ D. Sandanayaka, Y. Araki, P.^^A. Karr, J. Labuta, F. D’Souza, O. Ito, C.^^E. Anson, A.^^K. Powell, K. Ariga, J. Phys. Chem. C 2008, 112, 10559--10572</jnl>.

$<$ lit44><jnl>J. Labuta, S. Ishihara, T. Šikorský, Z. Futera, A. Shundo, L. Hanyková, J.^^V.

Burda, K. Ariga, J.^^P. Hill, Nat. Commun. 2013, 4, 3188</jnl>.

$<$ lit45 ><jnl>A. Shundo, S. Ishihara, J. Labuta, Y. Onuma, H. Sakai, M. Abe, K. Ariga, J.^^P. Hill, Chem. Commun. 2013, 49, 6870--6872</jnl>.

$<$ lit46><jnl>J. Labuta, Z. Futera, S. Ishihara, H. Kouřilová, Y. Tateyama, K. Ariga, J.^^P.

Hill, J. Am. Chem. Soc. 2014, 136, 2112--2118</jnl>.

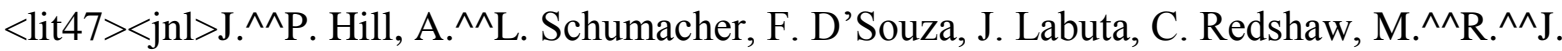

Elsegood, M. Aoyagi, T. Nakanishi, K. Ariga, Inorg. Chem. 2006, 45, 8288-$8296</$ jnl $>$.

$<$ lit48><jnl >M.^^J. Plater, S. Aitken, G. Bourhill, Tetrahedron, 2002, 58, 2405--2413</jnl $>$. $<$ lit49><jnl>C. ${ }^{\wedge \wedge}$ A. Martin, D. Ding, H. ${ }^{\wedge}$ S..$^{\wedge}$ J. van der Zant, J.^^M. van Ruitenbeek, New J. Phys. 2008, 10, 065008</jnl >.

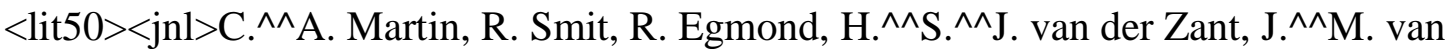
Ruitenbeek, Rev. Sci. Instrum. 2011, 82, 053907</jnl>.

$<$ lit51 $><$ jnl $>$ R. Frisenda, M.^^L. Perrin, H. Valkenier, J. Hummelen, H. ${ }^{\wedge}$ S. ${ }^{\wedge}$ J. van der Zant, Phys. Status Solidi B 2013, 250, 2431--2436</jnl>.

$<$ lit52 $><$ jnl $>$ J.^^S. Meisner, M. Kamenetska, M. Krikorian, M.^^L. Steigerwald, L.

Venkataraman, C.^^A. Nuckolls, Nano Lett. 2011, 11, 1575--1579</jnl>. $<$ lit53><jnl>P.^^E. Kornilovitch, A.^^M. Bratkovsky, Phys.Rev. B, 2001, 64, 195413</jnl>. $<$ lit54><jnl >W. Haiss, C. Wang, I. Grace, A. ${ }^{\wedge \wedge}$ S. Batsanov, D. ${ }^{\wedge} J$. Schiffrin, S.^^J. Higgins,

M.^^R. Bryce, C.^^J. Lambert, R.^^J. Nichols, Nat. Mater. 2006, 5, 995--1002</jnl>. $<$ lit55><other >Gaussian 09, Revision B.01, M.^^J. Frisch, G.^^W. Trucks, H.^^B. Schlegel, G. ${ }^{\wedge}$ E. Scuseria, M.^^A. Robb, J.^^R. Cheeseman, G. Scalmani, V. Barone, B. 
Mennucci, G. ${ }^{\wedge}$ A. Petersson, H. Nakatsuji, M. Caricato, X. Li, H.^^P. Hratchian, A.^^F. Izmaylov, J. Bloino, G. Zheng, J.^^L. Sonnenberg, M. Hada, M. Ehara, K. Toyota, R. Fukuda, J. Hasegawa, M. Ishida, T. Nakajima, Y. Honda, O. Kitao, H. Nakai, T. Vreven, J.^^A. Montgomery, Jr., J.^^E. Peralta, F. Ogliaro, M. Bearpark, J. ${ }^{\wedge} J$. Heyd, E. Brothers, K.^^N. Kudin, V.^^N. Staroverov, T. Keith, R. Kobayashi, J. Normand, K. Raghavachari, A. Rendell, J.^^C. Burant, S.^^S. Iyengar, J. Tomasi, M. Cossi, N. Rega, J.^^M. Millam, M. Klene, J.^^E. Knox, J.^^B. Cross, V. Bakken, C. Adamo, J. Jaramillo, R. Gomperts, R.^^E. Stratmann, O. Yazyev, A.^^J. Austin, R. Cammi, C. Pomelli, J.^^W. Ochterski, R.^^L. Martin, K. Morokuma, V.^^G. Zakrzewski, G. ${ }^{\wedge}$ A. Voth, P. Salvador, J.^^J. Dannenberg, S. Dapprich, A. ${ }^{\wedge} D$. Daniels, O. Farkas, J.^^B. Foresman, J.^^V. Ortiz, J. Cioslowski, D.^^J. Fox, Gaussian, Inc., Wallingford CT, 2010</other>.

Figure $^{\wedge} 1 \quad$ (a) Chemical structures of the compounds 1--3 used in this work with compound 2 also shown in 3-D projection. (b) Schematic of the mechanically controlled break junction set-up.

Figure $^{\wedge \wedge}$ Two-dimensional conductance histogram built from consecutive breaking traces recorded after drop-casting solutions containing the molecules under examination: a) OxP 2, b) reference compound 3 . The 1,650 and 10,000 breaking traces have been logarithmically binned in the conductance axis with 32 and 45 bins/decade respectively, and linearly in the displacement axis with 90 bins/nm respectively. The applied biases were 0.3 and $0.1^{\wedge \wedge} \mathrm{V}$ and the electrode speeds were 0.4 and $1.2^{\wedge \wedge} \mathrm{nm} / \mathrm{s}$ respectively.

Figure^^3 Individual breaking traces in presence of a molecule of: a) OxP 2: (i) empty junction, (ii) typical 'downhill' trace, (iii) single step plateau, (iv) double step plateau. b) (ivi) Selected traces for reference compound 3. The traces are respectively offset along the $x-$ axis by 1.2 and $1^{\wedge \wedge} \mathrm{nm}$ for clarity.

Figure $^{\wedge} 4$ (a) Macrocyclic moieties taken from the X-ray crystal structures of different OxP derivatives: (i) unsubstituted, ${ }^{[25]}$ (ii) $\mathrm{N}_{21}, \mathrm{~N}_{23}$-bis(4-bromobenzyl) (this work), (iii) 
2,3,7,8,12,13,16,17-octabromo- $\mathrm{N}_{21}, \mathrm{~N}_{23}$-bis(4-bromobenzyl). ${ }^{[28]}$ Angles $(\alpha)$ given are those subtended between the planes of opposing N-substituted pyrrole groups. (b) Geometry optimization of $\mathrm{N}_{21}, \mathrm{~N}_{23}$-disubstituted-OxP (i) unconstrained (distance between phenyls $\mathrm{C}_{1}$ '$\mathrm{C}_{1}{ }^{\prime}=13.15^{\wedge \wedge} \AA$ ), (ii) constrained. ( $\alpha$ again denotes angle between $\mathrm{N}$-substituted pyrroles with distance $\left.\mathrm{C}_{1}{ }^{\prime}-\mathrm{C}_{1}{ }^{\prime}=23.83^{\wedge \wedge} \AA\right)$.

Figure $^{\wedge \wedge 5}$ Schematic illustration of the mechanism of break junction formation for OxP, 2. (a) Initial ditopic adsorption of the molecule. (b) Break junction formation followed by (c), (d) molecular extension with increasing electrode separation. 